\section{(2) \\ BRAZIILIAN JOURNAL \\ OF MEDICAL AND BIOLOGICAL RESEARCH}

www.bjournal.com.br
ISSN 0100-879X

Volume 45 (6) 473-564 June 2012

BIOMEDICAL SCIENCES

AND

CLINICAL INVESTIGATION

Braz J Med Biol Res, May 2012, Volume 45(6) 488-496

doi: $10.1590 / \mathrm{S} 0100-879 X 2012007500033$

Pulsed ultrasound therapy accelerates the recovery of skeletal muscle damage induced by Bothrops jararacussu venom

J. Saturnino-Oliveira, M.A. Tomaz, T.F. Fonseca, G.A. Gaban, M. Monteiro-Machado, M.A. Strauch, B.L. Cons, S. Calil-Elias, A.M.B. Martinez and P.A. Melo

The Brazilian Journal of Medical and Biological Research is partially financed by

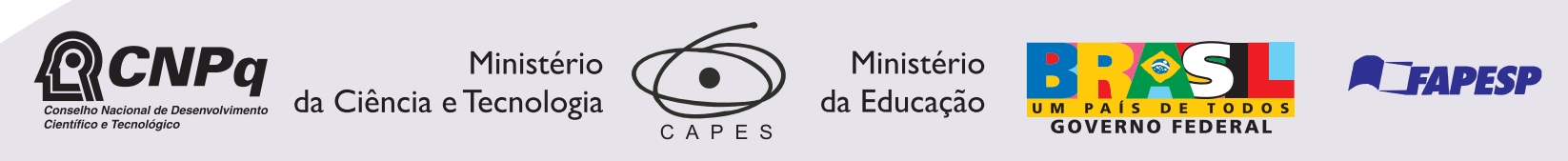

Institutional Sponsors

Scie/O
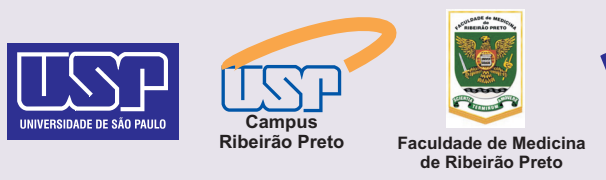

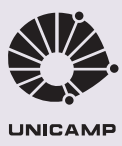

SHIMADZU

Associaçäo de Incentivo
à Pesquisa

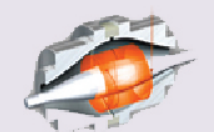




\title{
Pulsed ultrasound therapy accelerates the recovery of skeletal muscle damage induced by Bothrops jararacussu venom
}

\author{
J. Saturnino-Oliveira ${ }^{2,4 *}$, M.A. Tomaz ${ }^{1 *}$, T.F. Fonseca ${ }^{1}$, G.A. Gaban ${ }^{1}$, \\ M. Monteiro-Machado ${ }^{1}$, M.A. Strauch ${ }^{1}$, B.L. Cons ${ }^{1}$, S. Calil-Elias ${ }^{3}$, \\ A.M.B. Martinez $z^{2}$ and P.A. Melo ${ }^{1}$ \\ ${ }^{1}$ Programa de Farmacologia e Química Medicinal, Instituto de Ciências Biomédicas, \\ Universidade Federal do Rio de Janeiro, Rio de Janeiro, RJ, Brasil \\ ${ }^{2}$ Programa de Ciências Morfológicas, Instituto de Ciências Biomédicas, \\ Universidade Federal do Rio de Janeiro, Rio de Janeiro, RJ, Brasil \\ ${ }^{3}$ Departamento de Farmácia e Administração Farmacêutica, Faculdade de Farmácia, \\ Universidade Federal Fluminense, Niterói, RJ, Brasil \\ ${ }^{4}$ Departamento de Morfologia, Universidade Federal de Sergipe, Aracaju, SE, Brasil
}

\begin{abstract}
We studied the effect of pulsed ultrasound therapy (UST) and antibothropic polyvalent antivenom (PAV) on the regeneration of mouse extensor digitorum longus muscle following damage by Bothrops jararacussu venom. Animals (Swiss male and female mice weighing $25.0 \pm 5.0 \mathrm{~g} ; 5$ animals per group) received a perimuscular injection of venom (1 mg/kg) and treatment with UST was started $1 \mathrm{~h}$ later $\left(1 \mathrm{~min} / \mathrm{day}, 3 \mathrm{MHz}, 0.3 \mathrm{~W} / \mathrm{cm}^{2}\right.$, pulsed mode). Three and 28 days after injection, muscles were dissected and processed for light microscopy. The venom caused complete degeneration of muscle fibers. UST alone and combined with PAV $(1.0 \mathrm{~mL} / \mathrm{kg})$ partially protected these fibers, whereas muscles receiving no treatment showed disorganized fascicules and fibers with reduced diameter. Treatment with UST and PAV decreased the effects of the venom on creatine kinase content and motor activity (approximately 75 and $48 \%$, respectively). Sonication of the venom solution immediately before application decreased the in vivo and ex vivo myotoxic activities (approximately 60 and $50 \%$, respectively). The present data show that UST counteracts some effects of $B$. jararacussu venom, causing structural and functional improvement of the regenerated muscle after venom injury.
\end{abstract}

Key words: Pulsed therapeutic ultrasound; Bothrops snake venom; Myotoxicity; Mouse skeletal-muscle regeneration

\section{Introduction}

Muscle damage can be induced by many agents, e.g., extreme physical activity, trauma, specific disease states, chemical offenders, or the aging process (1). In tropical countries, a neglected cause of muscle damage is snakebite (2). The envenomation induces local effects such as acute edema, hemorrhage and myonecrosis, due to complex actions of various toxins present in the crude venoms (3). In severe cases, these local effects can lead to drastic sequelae such as permanent tissue loss, disability, or even amputation (4). Venoms of the genus Bothrops have been extensively studied under different protocols (5). Particularly, the venom of Bothrops jararacussu causes local myonecrosis due to the presence of components with proteolytic and phospholipase activities (6-10). Phospholipases present in $B$. jararacussu venom include two myotoxins: bothropstoxin I (Lys49 phospholipase $A_{2}$, $P L A_{2}$ ), which lacks enzymatic activity, and bothropstoxin II (Asp49 PLA 2$)$, which has catalytic activity $(7,11,12)$. The exact mechanism of myonecrosis induced by $\mathrm{PLA}_{2}$ is not fully understood, but its pathogenesis has several features in common with myonecrosis induced by other types of muscle damage: plasma membrane depolarization and disruption, sarcoplasmic reticulum retraction, clumped myofibrils, mitochondrial edema and rupture, and nucleus

Correspondence: P.A. Melo, Laboratório de Farmacologia das Toxinas, ICB, CCS, Universidade Federal do Rio de Janeiro, 21941-590 Rio de Janeiro, RJ, Brasil. Fax: +55-21-2610-3726. E-mail: melo.pa@gmail.com

*These authors contributed equally to this study.

Received September 28, 2011. Accepted February 17, 2012. Available online March 16, 2012. Published June 4, 2012. 
pyknosis $(11,13-16)$.

The currently available treatment for envenomation with $B$. jararacussu venom is the administration of antibothropic polyvalent antivenom (PAV), which has low and limited effectiveness against myotoxic activity $(9,10,12)$. PAV may prevent death, but does not prevent local tissue damage and subsequent functional disabilities (17). Researchers have been seeking alternative treatments that could antagonize such local effects, increasing tissue recovery and hastening the patient's return to daily routine (18-23).

Therapeutic ultrasound has been shown to induce biological activities related to tissue recovery, such as stimulation of collagen synthesis (24), activation of fibroblasts (25), increased blood flow (26), synthesis and/or release of growth factors and satellite cell activation, proliferation, and differentiation (27-29). Rather than continuous ultrasound with its heating effect, the pulsed mode has been used for muscle regeneration $(30,31)$. Delivered in pulsed, nonthermal settings, ultrasound can result in therapeutic effects through mechanical mechanisms, such as cavitation and acoustic streaming (32). In the present study, we determined if ultrasound would improve skeletal muscle regeneration following envenomation. We examined the effect of pulsed ultrasound therapy (UST) on mice following perimuscular injection of crude $B$. jararacussu venom.

\section{Material and Methods}

\section{Material}

B. jararacussu venom and the antibothropic PAV were provided by Instituto Vital Brazil, Rio de Janeiro, Brazil. This polyvalent antivenom is prepared from immunized horse serum, with $B$. alternatus, $B$. cotiara, $B$. jararaca, $B$. jararacussu, B. moojeni, $B$. neuwieddi, and $B$. pradoi; $1.0 \mathrm{~mL}$ antivenom neutralizes $2.5 \mathrm{mg}$ venom. The diagnostic kit used to determine creatine kinase (CK) activity was purchased from Bioclin-Brazil. All other reagents were of analytical grade. The ultrasound device used was the SONOCEL dual from BIOSET Industry of Electronics Technology (Brazil). The rotarod was purchased from AVS Special Projects (Brazil).

Adult male and female Swiss mice $(25.0 \pm 5.0 \mathrm{~g})$ were used. All the protocols were approved by the Ethics Committee on the Use of Animals of the Federal University of Rio de Janeiro.

\section{Experimental protocols}

Mice were divided into four groups of 4-6 animals. They were anesthetized with ethyl-ether and then injected with crude venom $(1.0 \mathrm{mg} / \mathrm{kg}$ in saline by applying $50 \mu \mathrm{L}$ of the solution to the extensor digitorum longus (EDL) muscle of the right limb (EDL perimuscular injection, in order to prevent direct mechanical damage to the muscle), as described previously $(9,10,33)$. All groups except the first, which was used as an untreated positive control, were treated $1 \mathrm{~h}$ after venom injection with UST under the following conditions: 1 $\mathrm{min} /$ day, frequency of $3 \mathrm{MHz}$, intensity of $0.3 \mathrm{~W} / \mathrm{cm}^{2}$, pulsed mode, $20 \%$ duty cycle ( $2 \mathrm{~ms}$ on, $8 \mathrm{~ms}$ off), pulse frequency of $100 \mathrm{~Hz}$. The effective radiating area was $1 \mathrm{~cm}^{2}$. To couple the ultrasound device to the mouse limb we used aqueous gel, with minimum pressure to maintain contact. One group received the same ultrasound treatment as the other groups, plus one intravenous injection of PAV $(1.0 \mathrm{~mL} / \mathrm{kg}) 15 \mathrm{~min}$ after venom injection. The negative control group received an injection of saline in the right limb.

\section{Measurement of muscle total CK content}

The total CK content of EDL muscle was measured in all animal groups. Mice were anesthetized with ethyl ether and killed by cervical dislocation 1, 3, 7, and 28 days after injection of $1.0 \mathrm{mg} / \mathrm{kg}$ venom. EDL muscles were dissected, freed from fat and tendons, dried and weighed. The muscles were then homogenized in $2 \mathrm{~mL}$ saline/0.1\% albumin, CK content was determined and reported as units per gram muscle tissue $(\mathrm{U} / \mathrm{g})(34)$.

\section{Histological examination}

Three and 28 days after perimuscular EDL injection of $1.0 \mathrm{mg} / \mathrm{kg}$ venom, the mice were anesthetized with ethyl ether and killed by cervical dislocation. The EDL muscles were dissected and fixed overnight in $2.5 \%$ glutaraldehyde and $4 \%$ paraformaldehyde in $0.1 \mathrm{M}$ sodium cacodylate buffer, $\mathrm{pH}$ 7.4. Next, they were washed three times in the same buffer and postfixed for $1 \mathrm{~h}$ in $1 \% \mathrm{OsO}_{4}$. The tissue was then dehydrated in increasing acetone concentrations (30-100\%) and embedded in Polybed 812 resin. Sections (500 nm) for light microscopy examination were obtained using an RMC ultramicrotome, stained with $1 \%$ toluidine blue, and then observed and photographed under light microscopy. Some mice injected with $1.0 \mathrm{mg} / \mathrm{kg} B$. jararacussu venom were killed after $24 \mathrm{~h}$ and their EDL muscles were isolated, fixed in standard paraformaldehyde, embedded in paraffin, sectioned, and stained with hematoxylin and eosin (HE).

\section{Edematogenic activity}

Five minutes after venom injection $(0.1,0.3,1.0$, and $3.0 \mathrm{mg} / \mathrm{kg}$ ), the animals received ultrasound treatment. The negative control group received only the injection of saline in the right limb. Measurements were made at 0, 15, 30, 60, and 90 min after venom injection or ultrasound treatment. An analog caliper was used to measure the mediolateral and anteroposterior widths of the paw, and the product of these values is reported as $\mathrm{mm}^{2}$.

\section{Leukocyte counts}

Two groups of four mice each received a perimuscular EDL injection of $1.0 \mathrm{mg} / \mathrm{kg} B$. jararacussu venom. Five minutes after venom injection, one group received an ultrasound application according to the protocol. Blood was collected by periorbital puncture before the injections and 2 and $24 \mathrm{~h}$ after them. Total and differential leukocyte counting was performed. 
A $10-\mu \mathrm{L}$ aliquot of blood was used to determine the total leukocyte count. Residual red blood cells were lysed by adding Turk's solution. For cytological examination, preparations were stained with May-Grunwald-Giemsa stain. Cell differentiation was performed by counting 100 cells, which were classified as lymphocytes, neutrophils or monocytes based on normal morphological criteria.

\section{Motor functional activity: rotarod test}

Motor activity was assessed using the rotarod test to analyze the riding time as previously described (35). The mice were trained daily for a period of $120 \mathrm{~s}$ for 5 days on the rotating cylinder ( $8 \mathrm{rpm})$. One, 3, 7, and 28 days after injection of $1.0 \mathrm{mg} / \mathrm{kg}$ venom alone or with treatments, the animals were submitted to the rotarod test and the time spent by the animal on the apparatus was recorded. UST was applied each day to the treatment groups, including a first application 5 min after venom injection. Each animal underwent three trials, and the mean time spent on the rod was determined for each group.

\section{In vivo and ex vivo B. jararacussu myotoxic activities}

The myotoxicity of $B$. jararacussu venom was studied both in vivo and ex vivo on the basis of CK activity. The ex vivo analysis employed observation of the rate of CK release from isolated mouse EDL muscle bathed in a solution containing the venom $(25 \mu \mathrm{g} / \mathrm{mL})$. Four EDL muscles were mounted vertically in a cylindrical chamber and superfused continuously with Ringer's solution equilibrated with 95\% $\mathrm{O}_{2} / 5 \% \mathrm{CO}_{2}$. At 30-60-min intervals, the solution perfusing the muscles was collected and replaced with fresh solution. The collected samples were used for the measurement of CK activity. Muscles were weighed at the end of the experiment. Enzyme activity is reported as international units corrected for muscle mass, where $1 \mathrm{U}$ is the amount that catalyzes the transformation of $1 \mu \mathrm{M}$ substrate at $25^{\circ} \mathrm{C}(36,37)$. The fresh solution for bathing the muscles contained either normal $B$. jararacussu venom or the venom solution after being sonicated with therapeutic ultrasound for $1 \mathrm{~min}$ at the same settings used so far, in order to observe the direct effect of ultrasound on venom myotoxic activity.

For the in vivo studies, 4 mice were injected with $50 \mu \mathrm{L}$ venom $(1.0 \mathrm{mg} / \mathrm{kg}), \mathrm{im}$, into the right thigh. Previous studies have shown that intramuscular injection of $0.1 \mathrm{~mL}$ saline has no effect on plasma CK levels $(36,37)$. Two hours after injection, the mice were anesthetized with ethyl ether for collection of blood samples (50-100 $\mu \mathrm{L})$ by orbital puncture. The plasma was separated by centrifugation and used for determination of CK activity. In vivo myotoxic activity was also observed with sonicated venom.

\section{SDS-PAGE}

The proteins present in the crude $B$. jararacussu venom were examined by electrophoresis after the venom was sonicated for 1 or 2 min. Polyacrylamide gel (12\%) electrophoresis in the presence of $0.1 \%$ sodium dodecyl sulfate (SDS-PAGE) was performed according to the standard method of Laemmli (38), and the proteins were stained with $0.1 \%$ Coomassie brilliant blue.

\section{Statistical analysis}

Data were analyzed statistically by the Student $t$-test; ANOVA was used for repeated measures and the Tukey test was used for group comparisons. Data are reported as means \pm SEM. Differences were considered to be statistically significant when $\mathrm{P}<0.05$.

\section{Results}

\section{Functional activity and edematogenic effect of the venom}

After $B$. jararacussu venom injection, all animals, including those receiving UST, showed a decrease in functional ability to stand on the rotarod. However, on the first and third day after injection, mice receiving venom only or venom + PAV showed a more pronounced decrease compared to animals treated with UST or UST + PAV. This result shows that UST decreased the impact of venom on motor functional activity. On the seventh day, all animals were able to remain on the rod for as long a time as the control mice, showing recovered function (Figure 1).

The venom induced dose-dependent edema (Figure

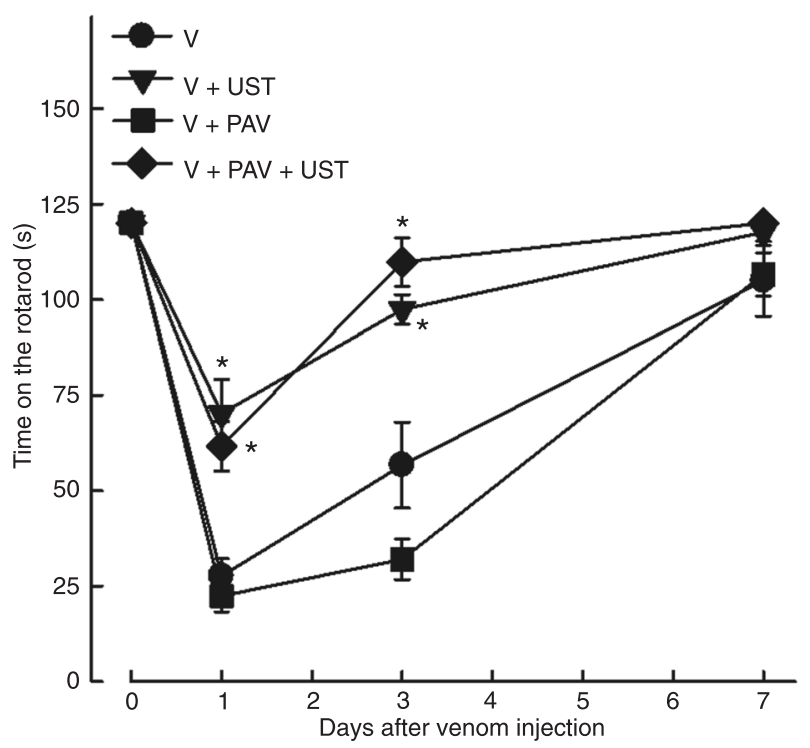

Figure 1. Rotarod activity. Time spent by mice on the rotarod (grpm) before and after receiving $1.0 \mathrm{mg} / \mathrm{kg}$ Bothrops jararacussu venom injected alone $(\mathrm{V})$ or associated with different treatments. Time zero represents the data obtained with each group before intramuscular venom injection or the treatments with pulsed therapeutic ultrasound (UST; $1 \mathrm{~min} /$ day) or polyvalent antivenom (PAV; $1.0 \mathrm{~mL} / \mathrm{kg}$ ), or PAV + UST. Data are reported as means \pm $\operatorname{SEM}(\mathrm{N}=6) .{ }^{*} \mathrm{P}<0.05$ vs venom group (ANOVA). 

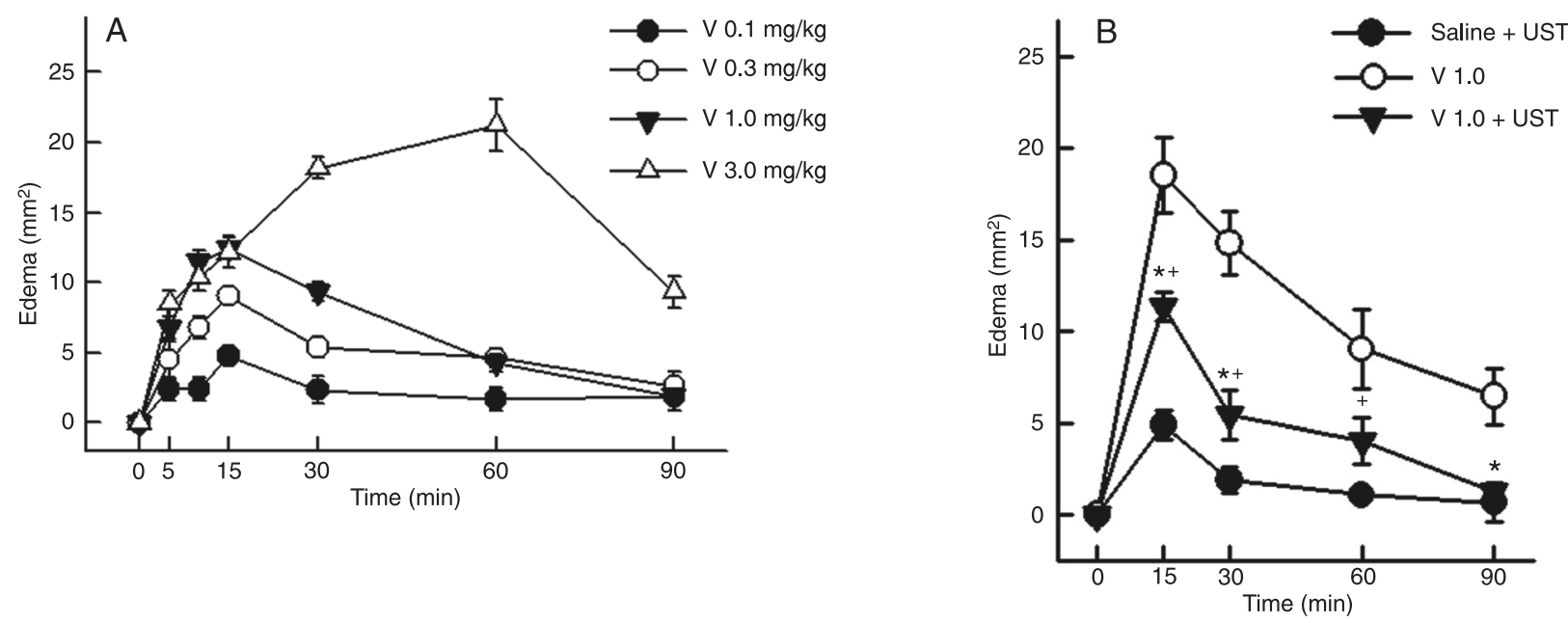

Figure 2. Edematogenic activity. $A$, Edema induced by intramuscular injection of different doses of Bothrops jararacussu venom (V). B, Effect of pulsed therapeutic ultrasound (UST) on the edematogenic activity of $1.0 \mathrm{mg} / \mathrm{kg} B$. jararacussu venom. UST was applied $5 \mathrm{~min}$ after venom injection. Data are reported as means \pm SEM $(N=5)$. ${ }^{*} P<0.05$ compared to $V+U S T ;{ }^{+} P<0.05$ for the $V+U S T$ group compared to the control group (saline + UST; ANOVA).

$2 \mathrm{~A}$ ), reaching a peak within 15 min with lower doses, and after about 60 min with the highest dose. When applied $5 \mathrm{~min}$ after $1.0 \mathrm{mg} / \mathrm{kg}$ venom administration, UST decreased the edematogenic effect, suggesting that reduced edema can improve the motor function of mice receiving UST (Figure $2 \mathrm{~B}$ ).

\section{Changes in CK content}

Damage to the sarcolemma, allowing $\mathrm{CK}$ to leave the cell, was marked, so that CK content inside the cell dropped substantially after perimuscular venom injection (Figure 3). This effect was reversed by UST. One day after venom injection, EDL CK content was 41.05\% of the CK content observed in the group receiving only saline. The treatments with UST, PAV and PAV + UST reduced the drop in CK content induced by the action of the venom (Figure 3A). On the third day, the CK content was reduced to nearly $33 \%$

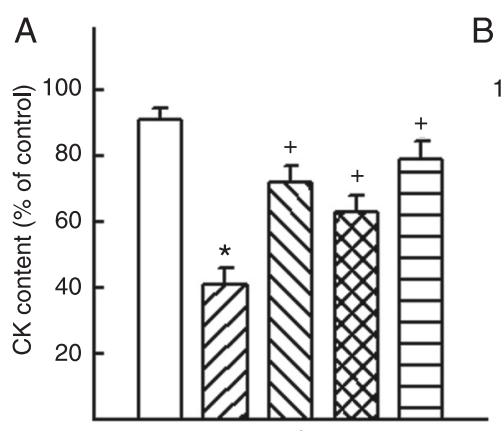

B
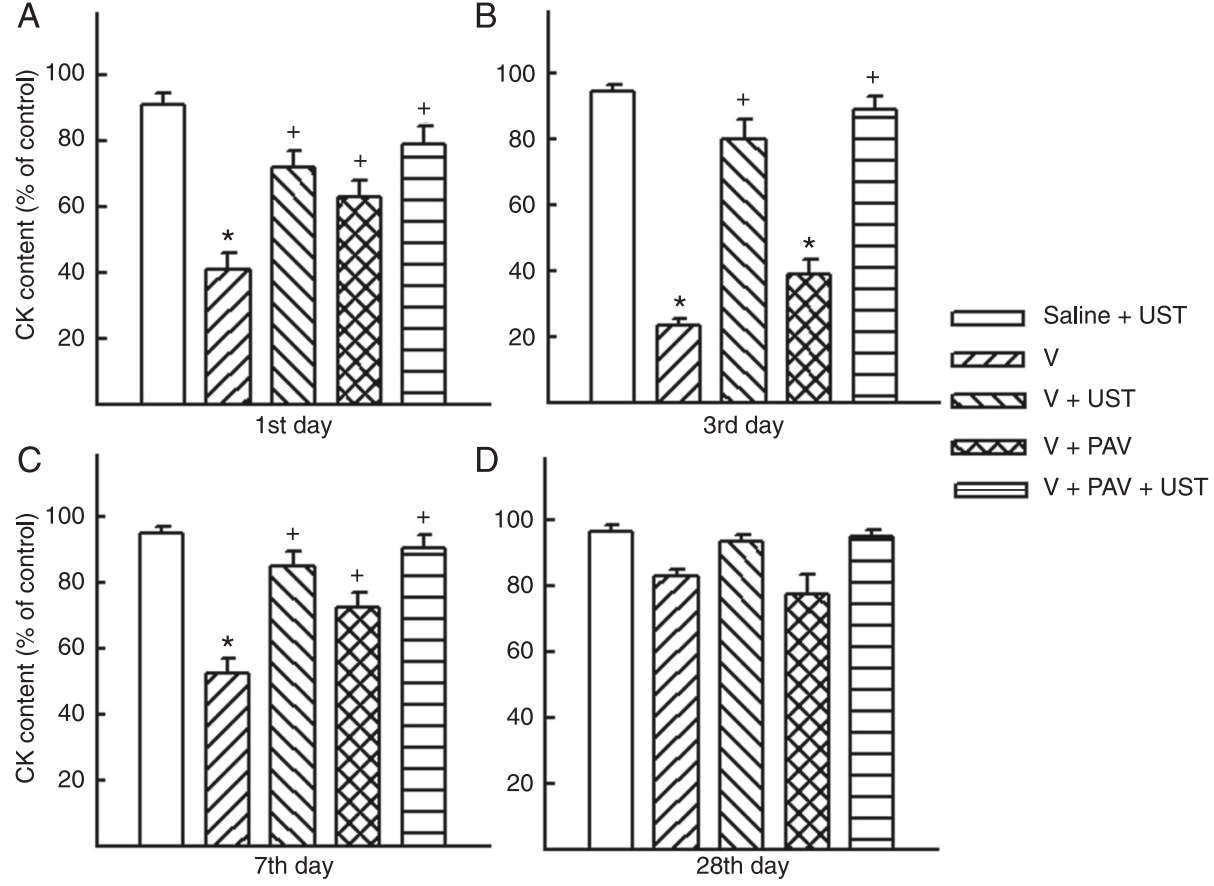

Figure 3. Creatine kinase (CK) content of mouse extensor digitorum longus muscle after perimuscular injection of Bothrops jararacussu venom (V;1.0 mg/kg) alone and treatment with pulsed therapeutic ultrasound (UST) or polyvalent antivenom (PAV, $1.0 \mathrm{~mL} / \mathrm{kg}$ ) or UST + PAV. Data are reported as the percentage of CK 1, 3, 7, and 28 days after venom injection and the specific treatments. The control group was taken to be $\mathrm{CK}$ content $=100 \%$ (data not shown). Panels $A$ and $B$ show the drop in CK content 1 and 3 days after venom injection, respectively. Note the more pronounced effect of the venom on the third day, and how PAV alone failed to prevent this effect, while UST alone or UST + PAV protected the muscles. By day 28 all groups showed CK content near control values. Data are reported as means \pm SEM $(N=5)$. * $P<0.05$ compared to control (saline + UST); ${ }^{+} P<0.05$ compared to the venom group (ANOVA). 
in the venom group, PAV did not decrease the effect of the venom, while the treatments with UST and UST + PAV did protect the muscle from CK loss (Figure 3B). The CK content was restored in the treated groups 7 days after venom injection, but the untreated group recovered only partially (52.6\% of control) (Figure 3C). Twenty-eight days after venom injection and treatments, all groups had a CK content similar to that of the control group (Figure 3D).

\section{Histological results}

Twenty-four hours after perimuscular injection of $B$. jararacussu venom, the longitudinal section of HE-stained EDL muscle showed an intense inflammatory infiltrate and myofibers in the process of necrosis (Figure 4). Figure 5 shows light micrographs of EDL muscle 3 days after venom injection and treatments ( $N=5$ per group). In the muscles that received saline injection and UST we observed a typical structural organization of skeletal muscle (Figure $5 A$ and $B$ ), showing that UST does not damage muscle cells. Animals receiving only venom showed degenerated peripheral cells (Figure 5C). Treatment with UST partially protected the cells from the action of the venom because the damaged area was smaller than in the venom group (Figure 5D). Muscles treated with PAV combined with UST showed a normal profile, indicating protection from venom, while PAV alone was ineffective (Figure 5E and F).

Twenty-eight days after injection, all the animals (venom only and treatments) had EDL muscle profiles resembling the architecture of the control group (Figure 6). Peripheral cells were arranged in fascicles, showing regenerated cells with their characteristic centralized nuclei (Figure 6D).

\section{Changes in leukocyte counts}

Venom induced a marked decrease in total leukocyte count 2 $\mathrm{h}$ after injection (Figure 7A). The

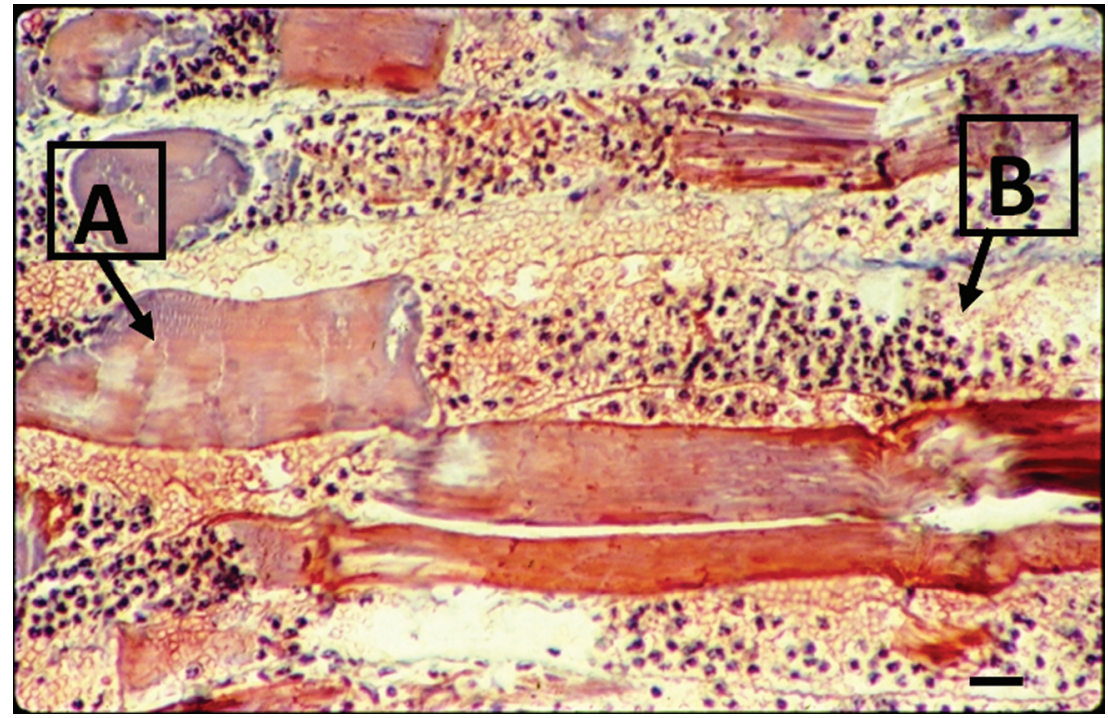

Figure 4. Light microscopy of mouse extensor digitorum longus muscle $24 \mathrm{~h}$ after perimuscular injection of $1.0 \mathrm{mg} / \mathrm{kg}$ Bothrops jararacussu venom. Longitudinal section stained with hematoxylin and eosin. Note necrotic myofibers in different stages of degeneration, as shown in $A$, and the intense acute inflammatory infiltrate in $B$ (bar $=10 \mu \mathrm{m})$.
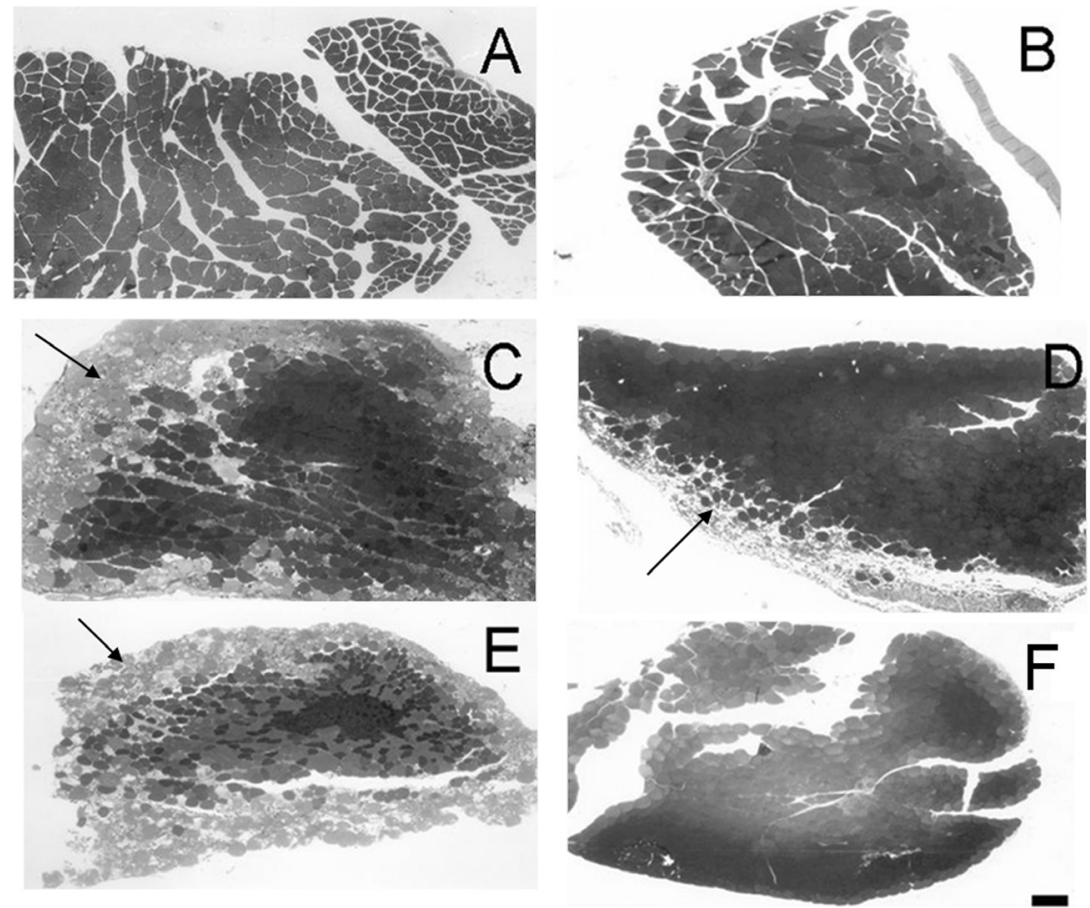

Figure 5. Cross sections of extensor digitorum longus muscles 3 days after receiving $(A)$ saline and $(B)$ saline + pulsed therapeutic ultrasound (UST); $(C)$ venom $(\mathrm{V}, 1.0 \mathrm{mg} / \mathrm{kg})$, and venom plus treatments: $(D) \mathrm{V}+\mathrm{UST} ;(E) \mathrm{V}+\mathrm{PAV} ;(F) \mathrm{V}+\mathrm{PAV}+\mathrm{UST}(\mathrm{N}=5$ per group). $A$, Panoramic view of control muscle showing normal cells with no morphological changes, which was not altered with UST $(B)$. C-E, Note the damage to peripheral muscle fibers (arrows) and the disorganization of the bundles, while central fibers remain intact, although in $D$ the damage to peripheral fibers is far less pronounced than in $C$ and $E$. $F$, Observe no damage to peripheral fibers when UST was used in addition to PAV $(\mathrm{bar}=170 \mu \mathrm{m})$. 

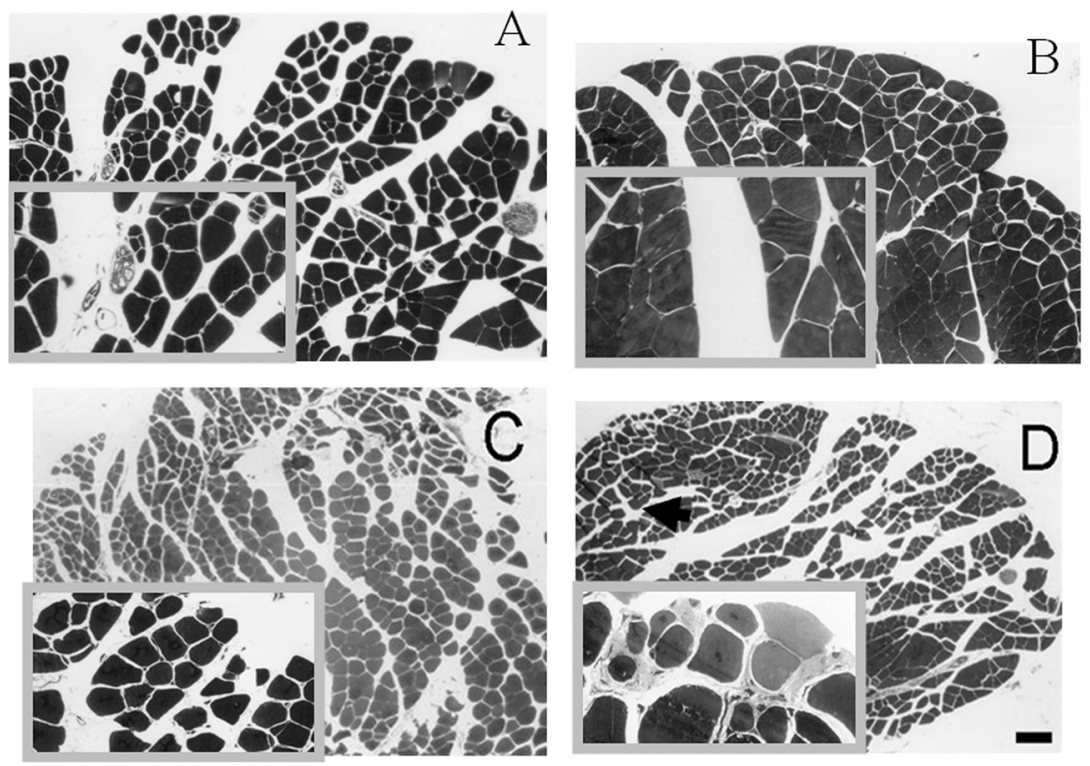

Figure 6. Cross sections of extensor digitorum longus muscles 28 days after receiving $(A)$ venom $(\mathrm{V}, 1.0 \mathrm{mg} / \mathrm{kg})$, and venom plus treatments: $(B) \mathrm{V}+$ pulsed therapeutic ultrasound (UST); (C) $\mathrm{V}+$ polyvalent antivenom (PAV); (D) V + PAV + UST. Note that muscle resembles the control, with recovered architecture, in particular the organized fibers and bundles, both centrally and peripherally. $D$, The insert shows fibers with centrally located nuclei (arrow) representing regenerated cells $(\mathrm{bar}=170 \mu \mathrm{m})$. Insert magnifications from original pictures: $A=2 \mathrm{X} ; B=3.5 \mathrm{X} ; C=2.5 \mathrm{X} ; D=5 \mathrm{X}$.
A

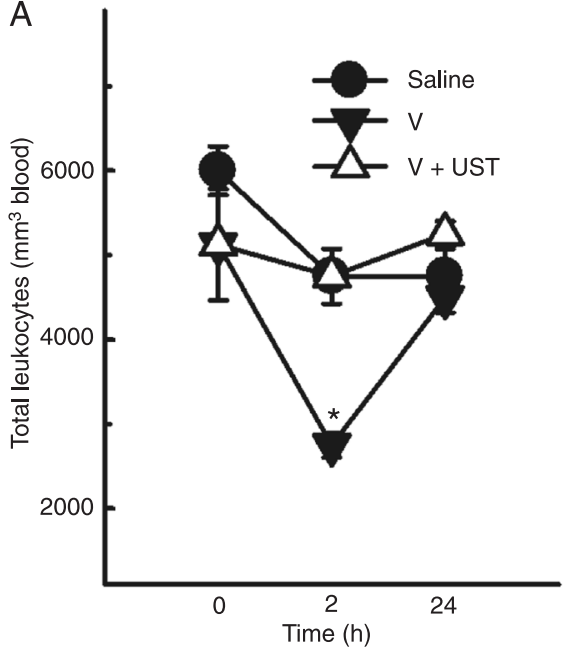

B

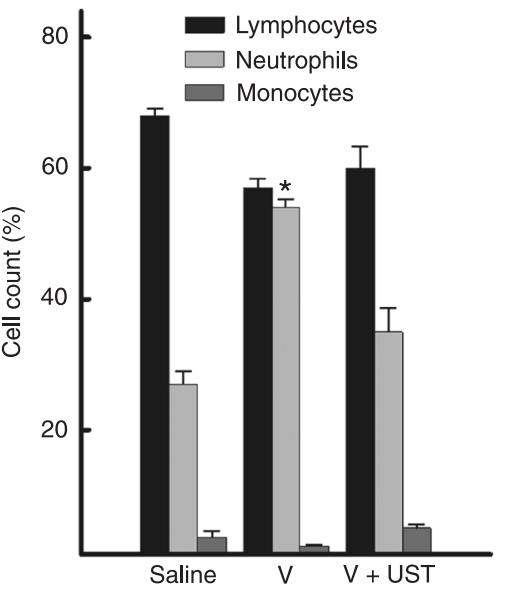

Figure 7. Total and differential leukocyte counts of mouse blood. A, Number of total leukocytes in mouse blood after intramuscular injection of $1.0 \mathrm{mg} / \mathrm{kg}$ Bothrops jararacussu venom $(\mathrm{V})$ alone or with treatment with pulsed therapeutic ultrasound (UST; $5 \mathrm{~min}$ after venom injection). Data are reported as means \pm SEM $(N=5)$. ${ }^{*} P<0.05$ for venom alone compared to $\mathrm{V}+\mathrm{UST}$ or saline (ANOVA). $B$, Differential leukocyte count $2 \mathrm{~h}$ after venom injection and treatment with UST. Data are reported as means \pm SEM $(N=5)$. ${ }^{*} P<0.05$ for venom alone compared to $\mathrm{V}+\mathrm{UST}$ or saline (ANOVA). There was no significant difference between saline and V + UST. leukocyte levels returned to the same range as that of the saline-injected group after $24 \mathrm{~h}$. UST prevented the total leukocyte count from decreasing. Figure 7B shows the differential leukocyte counts. The proportion of neutrophils increased after venom injection, an event that, again, was prevented by UST.

\section{In vivo and ex vivo CK activity}

$B$. jararacussu venom induced an increase in CK activity, both ex vivo (from $1.47 \pm 0.38$ to $40.01 \pm 1.85$ $\mathrm{U} \cdot \mathrm{g}^{-1} \cdot \mathrm{h}^{-1}$ after $90 \mathrm{~min}$ of exposure) and in vivo (from $81.73 \pm 22.14$ to $1808.98 \pm 285.15 \mathrm{U} / \mathrm{L}$ ) compared to control conditions, which consisted of exposure to saline (Figure 8A and B, respectively). Interestingly, application of UST directly to the venom solution prior to exposure caused a smaller increase in ex vivo (from $1.47 \pm 0.38$ to $27.60 \pm 2.75 \mathrm{U} \cdot \mathrm{g}^{-1} \cdot \mathrm{h}^{-1}$ ) and in vivo (from $81.73 \pm 22.14$ to $967.31 \pm 71.15$ U/L) CK activity compared to venom without ultrasound application, suggesting a direct effect on the toxins present in the crude venom. Surprisingly, the analysis of the protein profiles of the crude venom, assessed by SDS-PAGE electrophoresis, showed no effect of ultrasound on the protein contents (Figure 8C).

\section{Discussion}

We have shown that pulsed UST could ameliorate the damaging effects of $B$. jararacussu venom, from edema to myonecrosis, including motor dysfunction, in mice. Treatment with UST reduced the functional and edematogenic activity of the venom, while PAV did not. In parallel to decreasing edema and improving motor activity, UST was able to counteract the considerable drop in EDL CK content, as well as protecting peripheral fibers from necrosis. Therefore, mice receiving treatment with UST showed better muscle performance, i.e., because the morphological and biochemical properties seemed to be preserved, their physical activity was superior to 
that achieved by untreated animals. Although in morphological analysis we did not measure the extent of the damaged area or the number of centrally located nuclei, this qualitative demonstration of the positive effects of ultrasound was confirmed quantitatively by CK content and rotarod performance. The evaluation of PAV alone confirmed previous data showing it to be of limited effectiveness against myonecrosis induced by $B$. jararacussu venom (17). On the other hand, if applied in combination with UST, PAV seems to be of value.

Adult skeletal muscle is a notably stable tissue and has a remarkable ability for self-repair when damaged, irrespective of the underlying cause of damage. However, the regeneration process can be relatively slow, which obviously delays the return of human patients to their normal activity. Thus, research has been directed to discover ways to both improve and accelerate the process of muscle recovery.

Therapeutic ultrasound has been shown to affect a range of cellular activities that together contribute to hasten and enhance functional rehabilitation. Although therapeutic ultrasound is known for its heating capacity in deep tissues, it may have other effects on tissues that are not related to heat generation (39). Johns (32) proposed a molecular mechanism whereby the mechanical properties of pulsed ultrasound interact with molecular (and multimolecular) complexes within the cell, thereby modulating membrane properties, cell metabolism and synthesis of substances, specifically the activation of proteins and signal-transduction pathways, which may result in modifications of cellular function. Since these nonthermal effects of therapeutic ultrasound have been implicated in tissue regeneration, the pulsed mode has received great attention from researchers in this field $(30,31)$.

The observation in both ex vivo and in vivo analyses that direct sonication could decrease myotoxicity was surprising, suggesting that it could degrade protein in the venom. However, SDS-PAGE electrophoresis of the venom revealed that proteins present in the normal venom solution were still present in the solutions receiving UST, even when the duration of application was doubled. A possible explanation could be that ultrasound directly applied to the venom could disturb any active site of proteins that contribute to the myotoxic action of the venom, without changing the protein primary structure, which is responsible for the molecular weight. The frequency resonance hypothesis for the nonthermal effects of therapeutic ultrasound has been proposed (32). This hypothesis suggests that the mechanical energy within the US wave is absorbed by proteins, altering the 3-D structural conformation of an individual protein or the function of a multimolecular complex. Further studies will be necessary to clarify the specific effect
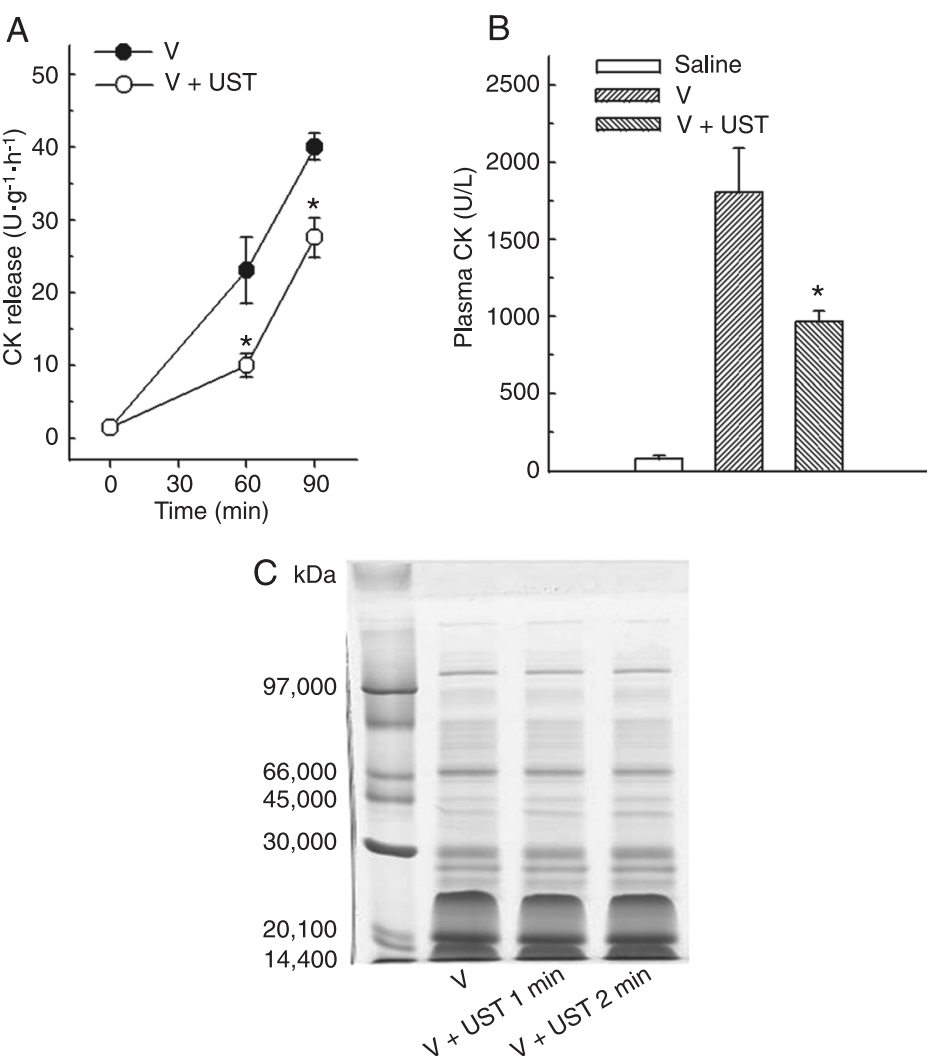

Figure 8. Ex vivo and in vivo myotoxic activities. $A$, Creatine kinase $(\mathrm{CK})$ elease from mouse extensor digitorum longus muscles following exposure olication directly to the venom solution prior to exposure. $B$, Plasma $\mathrm{kg}$ ) following ultrasound application to the venom solution. Data are reported as means \pm SEM $(\mathrm{N}=5)$. ${ }^{*} \mathrm{P}<0.05 \mathrm{vs}$ venom group (Student $t$-test). $C$, SDS-PAGE electrophoresis of $B$. jararacussu venom or the venom after ulasound application for 1 or $2 \mathrm{~min}$.

of ultrasound on the properties of the toxins.

Overall, our data suggest that pulsed ultrasound therapy can be useful in the management of skeletal muscle injury following $B$. jararacussu snake bite. Adding pulsed ultrasound therapy to the therapeutic arsenal against snake bites could contribute to hastening recovery, either by preventing venom action or improving recovery, or both. Prevention of the actions of the venom can be due to the resonance hypothesis regarding proteins, described above, or it can result from antiinflammatory effects of pulsed ultrasound. Improved recovery could come from one or more of the therapeutic ultrasound properties shown earlier in this section.

\section{Acknowledgments}

Research supported by FAPERJ, CNPq, CAPES, Fundação Universitária José Bonifácio (FUJB, UFRJ), and PRONEX. 


\section{References}

1. Close GL, Kayani A, Vasilaki A, McArdle A. Skeletal muscle damage with exercise and aging. Sports Med 2005; 35: 413427.

2. White J. Bites and stings from venomous animals: a global overview. Ther Drug Monit 2000; 22: 65-68.

3. Rosenfeld G. Symptomatology, pathology and treatment of snake bites. In: Anonymous, South America. Venomous animals and their venoms. New York: Academic Press; 1971. p 345-384.

4. Lomonte B, Angulo Y, Calderon L. An overview of lysine-49 phospholipase A2 myotoxins from crotalid snake venoms and their structural determinants of myotoxic action. Toxicon 2003; 42: 885-901.

5. Gutierrez JM, Ownby CL. Skeletal muscle degeneration induced by venom phospholipases A2: insights into the mechanisms of local and systemic myotoxicity. Toxicon 2003; 42: 915-931.

6. Queiroz LS, Santo NH, Rodrigues-Simioni L, Prado-Franceschi J. Muscle necrosis and regeneration after envenomation by Bothrops jararacussu snake venom. Toxicon 1984; 22: 339-346.

7. Homsi-Brandeburgo MI, Queiroz LS, Santo-Neto H, RodriguesSimioni L, Giglio JR. Fractionation of Bothrops jararacussu snake venom: partial chemical characterization and biological activity of bothropstoxin. Toxicon 1988; 26: 615-627.

8. Anndriao-Escarso SH, Soares AM, Rodrigues VM, Angulo Y, Diaz C, Lomonte B, et al. Myotoxic phospholipases A(2) in bothrops snake venoms: effect of chemical modifications on the enzymatic and pharmacological properties of bothropstoxins from Bothrops jararacussu. Biochimie 2000; 82: 755-763.

9. Calil-Elias S, Martinez AM, Melo PA. Effect of heparin and antivenom on skeletal muscle damage produced by Bothrops jararacussu venom. Histol Histopathol 2002; 17: 463-470.

10. Calil-Elias S, Thattassery E, Martinez AM, Melo PA. Effect of perimuscular injection of Bothrops jararacussu venom on plasma creatine kinase levels in mice: influence of dose and volume. Braz J Med Biol Res 2002; 35: 1233-1235.

11. Gutierrez JM, Nunez J, Diaz C, Cintra AC, Homsi-Brandeburgo MI, Giglio JR. Skeletal muscle degeneration and regeneration after injection of bothropstoxin-II, a phospholipase A2 isolated from the venom of the snake Bothrops jararacussu. Exp Mol Pathol 1991; 55: 217-229.

12. Murakami MT, Vicoti MM, Abrego JR, Lourenzoni MR, Cintra $A C$, Arruda EZ, et al. Interfacial surface charge and free accessibility to the PLA2-active site-like region are essential requirements for the activity of Lys49 PLA2 homologues. Toxicon 2007; 49: 378-387.

13. Harris JB, Cullen MJ. Muscle necrosis caused by snake venoms and toxins. Electron Microsc Rev 1990; 3: 183-211.

14. Harris JB. Myotoxic phospholipases A2 and the regeneration of skeletal muscles. Toxicon 2003; 42: 933-945.

15. Harris JB, Vater R, Wilson M, Cullen MJ. Muscle fibre breakdown in venom-induced muscle degeneration. J Anat 2003; 202: 363-372.

16. Melo PA, Burns CF, Blankemeyer JT, Ownby CL. Membrane depolarization is the initial action of crotoxin on isolated murine skeletal muscle. Toxicon 2004; 43: 111-119.
17. da Silva NM, Arruda EZ, Murakami YL, Moraes RA, El-Kik CZ, Tomaz MA, et al. Evaluation of three Brazilian antivenom ability to antagonize myonecrosis and hemorrhage induced by Bothrops snake venoms in a mouse model. Toxicon 2007; 50: 196-205.

18. Melo PA, Homsi-Brandeburgo MI, Giglio JR, Suarez-Kurtz G. Antagonism of the myotoxic effects of Bothrops jararacussu venom and bothropstoxin by polyanions. Toxicon 1993; 31: 285-291.

19. Melo PA, do Nascimento MC, Mors WB, Suarez-Kurtz G. Inhibition of the myotoxic and hemorrhagic activities of crotalid venoms by Eclipta prostrata (Asteraceae) extracts and constituents. Toxicon 1994; 32: 595-603.

20. da Silva AJM, Melo PA, Silva NM, Brito FV, Buarque CD, de Souza DV, et al. Synthesis and preliminary pharmacological evaluation of coumestans with different patterns of oxygenation. Bioorg Med Chem Lett 2001; 11: 283-286.

21. da Silva AJ, Coelho AL, Simas AB, Moraes RA, Pinheiro DA, Fernandes FF, et al. Synthesis and pharmacological evaluation of prenylated and benzylated pterocarpans against snake venom. Bioorg Med Chem Lett 2004; 14: 431-435.

22. Arruda EZ, Silva NM, Moraes RA, Melo PA. Effect of suramin on myotoxicity of some crotalid snake venoms. Braz J Med Biol Res 2002; 35: 723-726.

23. Murakami MT, Lourenzoni MR, Arruda EZ, Tomaz MA, Vicoti $\mathrm{MM}$, Abrego JR, et al. Biochemical and structural investigations of Bothropstoxin-II, a myotoxic Asp49 phospholipase A2 from Bothrops jararacussu venom. Protein Pept Lett 2008; 15: 1002-1008.

24. Webster DF, Harvey W, Dyson M, Pond JB. The role of ultrasound-induced cavitation in the 'in vitro' stimulation of collagen synthesis in human fibroblasts. Ultrasonics 1980; 18: 33-37.

25. da Cunha A, Parizotto NA, Vidal Bde C. The effect of therapeutic ultrasound on repair of the achilles tendon (tendo calcaneus) of the rat. Ultrasound Med Biol 2001; 27: 16911696.

26. Noble JG, Lee V, Griffith-Noble F. Therapeutic ultrasound: the effects upon cutaneous blood flow in humans. Ultrasound Med Biol 2007; 33: 279-285.

27. Rantanen J, Thorsson O, Wollmer $\mathrm{P}$, Hurme $\mathrm{T}$, Kalimo $\mathrm{H}$. Effects of therapeutic ultrasound on the regeneration of skeletal myofibers after experimental muscle injury. $A m \mathrm{~J}$ Sports Med 1999; 27: 54-59.

28. Karnes JL, Burton HW. Continuous therapeutic ultrasound accelerates repair of contraction-induced skeletal muscle damage in rats. Arch Phys Med Rehabil 2002; 83: 1-4.

29. Piedade MC, Galhardo MS, Battlehner CN, Ferreira MA, Caldini EG, de Toledo OM. Effect of ultrasound therapy on the repair of gastrocnemius muscle injury in rats. Ultrasonics 2008; 48: 403-411.

30. Freitas TP, Gomes M, Fraga DB, Freitas LS, Rezin GT, Santos PM, et al. Effect of therapeutic pulsed ultrasound on lipoperoxidation and fibrogenesis in an animal model of wound healing. J Surg Res 2010; 161: 168-171.

31. Wu S, Kawahara Y, Manabe T, Ogawa K, Matsumoto M, Sasaki A, et al. Low-intensity pulsed ultrasound accelerates osteoblast differentiation and promotes bone formation in an osteoporosis rat model. Pathobiology 2009; 76: 99-107. 
32. Johns LD. Nonthermal effects of therapeutic ultrasound: the frequency resonance hypothesis. J Athl Train 2002; 37: 293-299.

33. Melo PA, Ownby CL. Ability of wedelolactone, heparin, and para-bromophenacyl bromide to antagonize the myotoxic effects of two crotaline venoms and their PLA2 myotoxins. Toxicon 1999; 37: 199-215.

34. Melo PA, Ownby CL. Different sensitivity of fast- and slowtwitch muscles to some snake venoms and myotoxins. Toxicon 1996; 34: 653-669.

35. Ko MH, Chen WP, Lin-Shiau SY, Hsieh ST. Age-dependent acrylamide neurotoxicity in mice: morphology, physiology, and function. Exp Neurol 1999; 158: 37-46.
36. Melo PA, Suarez-Kurtz G. Release of creatine kinase from skeletal muscles by Bothrops venoms: heparin potentiation of inhibition by antivenin. Braz J Med Biol Res 1988; 21: 545-548.

37. Melo PA, Suarez-Kurtz G. Release of sarcoplasmic enzymes from skeletal muscle by Bothrops jararacussu venom: antagonism by heparin and by the serum of South American marsupials. Toxicon 1988; 26: 87-95.

38. Laemmli UK. Cleavage of structural proteins during the assembly of the head of bacteriophage T4. Nature 1970; 227 : 680-685.

39. ter Haar G. Therapeutic applications of ultrasound. Prog Biophys Mol Biol 2007; 93: 111-129. 